Para enlazar con este artículo / To link to this article:

https://doi.org/10.6035/MonTI.2020.12.10

Para citar este artículo / To cite this article:

Arias-Badia, Blanca \& Anna Fernández-Torné. (2020) "El experto en lenguaje fácil de comprender: un nuevo perfil educativo y profesional en el ámbito de la lengua española." En: Richart-Marset, Mabel \& Francesca Calamita (eds.) 2020. Traducción y Accesibilidad en los medios de comunicación: de la teoría a la práctica / Translation and Media Accessibility: from Theory to Practice. MonTI 12, pp. 295-312.

\title{
EL EXPERTO EN LENGUAJE FÁCIL DE COMPRENDER: UN NUEVO PERFIL EDUCATIVO Y PROFESIONAL EN EL ÁMBITO DE LA LENGUA ESPAÑOLA ${ }^{1}$
}

\author{
BLANCA ARIAS-BADIA \\ blanca.arias@uab.cat \\ Universitat Autònoma de Barcelona \\ ANNA FERNÁNDEZ-TORNÉ \\ anna.torne@gmail.com \\ Universitat Autònoma de Barcelona
}

\section{Resumen}

Las salidas profesionales de los estudiantes formados en lenguas se diversifican cada vez más en el mundo globalizado. Este artículo presenta los resultados de un cuestionario centrado en un perfil profesional emergente en el ámbito de la lengua española: el experto en la creación, revisión y adaptación de contenidos fáciles de comprender. Dicho perfil es el principal foco de interés del proyecto de innovación docente EASIT (Formación en Acceso Fácil para la Inclusión Social), el cual pretende describir este

1. Esta investigación forma parte del proyecto EASIT financiado por el programa Erasmus+ Strategic Partnerships for Higher Education (2018-1-ES01-KA203-05275) de la Comisión Europea. El apoyo de la Comisión Europea en esta publicación no constituye un apoyo de su contenido, el cual refleja únicamente la perspectiva de las autoras, y no puede responsabilizarse a la Comisión del uso que se dé a la información aquí recogida. Las autoras son miembros del grupo consolidado TransMedia Catalonia (2017SGR113). La participación de Blanca Arias-Badia en esta investigación ha recibido el apoyo de la Agencia Estatal de Investigación, en el marco del programa de ayudas posdoctorales Juan de la Cierva-Formación (FJCI-2017-32064). 
nuevo perfil para dotarlo de visibilidad y proponer para él un programa docente y materiales de formación específicos, hasta ahora escasos para quien quiera seguir esta vía de especialización.

Palabras clave: Lenguaje fácil de comprender; Lenguaje de lectura fácil; Lenguaje llano; EASIT; Accesibilidad.

\begin{abstract}
"The expert in easy-to-understand language: A new educational and professional profile in the field of the Spanish language"

The professional opportunities of students trained in languages are increasingly diversified in the globalized world. This article presents the results of a questionnaire focused on an emerging professional profile in the field of the Spanish language: the expert in the creation, revision and adaptation of easy-to-understand content. This profile is the main focus of interest of the teaching innovation project EASIT (Easy Access for Social Inclusion Training), which aims to describe this new profile to make it more visible and to propose a specific teaching program and training materials for it - until now, there is a poor academic offer for those who want to follow this path of specialization.
\end{abstract}

Keywords: Easy-to-understand language; Easy-to-read language; Plain language; EASIT; Accessibility.

\title{
1. Introducción
}

En 2006 se aprobó la Convención sobre los derechos de las personas con discapacidad de las Naciones Unidas (ONU), con el propósito de "promover, proteger y asegurar el goce pleno y en condiciones de igualdad de todos los derechos humanos y libertades fundamentales por todas las personas con discapacidad" (Artículo 1), incluidas las personas con discapacidades a largo plazo bien físicas, sensoriales, mentales o intelectuales. La aprobación de la Convención de la ONU dio lugar a la ampliación del marco normativo y legislativo en materia de accesibilidad, y a la consecuente regulación de las condiciones de accesibilidad y establecimiento de sanciones en caso de incumplimiento.

Para que las personas con discapacidad puedan ejercer plenamente sus derechos y participar en igualdad de condiciones en cualquier ámbito de la 
vida, es necesario tener presente o bien el diseño universal o bien la introducción de adaptaciones que se ajusten a las necesidades específicas de cada grupo. De este modo, se garantiza el acceso no solo al transporte y a los espacios en general, sino también a los productos, a los dispositivos, a la educación, a las comunicaciones y a la información, incluidos los medios de comunicación y las tecnologías de la información, así como a cualquier otro servicio en general.

Con este claro objetivo de acceso a la información nacieron la lectura fácil (LF) y el lenguaje llano (LL) como servicios de accesibilidad, y en particular, en el caso de la LF, de accesibilidad cognitiva destinado en primera instancia a personas con discapacidades intelectuales. No obstante, con el paso del tiempo se ha ampliado el abanico de destinatarios ideales de dichos servicios, puesto que se ha demostrado que también pueden beneficiarse de ellos en especial las personas mayores y las personas con poco dominio del idioma, entre otras.

Ya sea por una mayor concienciación o por imperativo legal, muchas son ya las organizaciones europeas que a nivel nacional o regional se dedican a ofrecer servicios de accesibilidad cognitiva, como, por ejemplo, Dyslexiförbundet (Suecia), Zavod RISA (Eslovenia), Associació Lectura Fàcil (Cataluña), Inclusion Europe (Bélgica), Plena Inclusión (España), Atempo (Austria), Me Itse ry (Finlandia), Nous Aussi y UNAPEI (Francia), Büro für Leichte Sprache de Lebenshilfe Bremen (Alemania), etc. Sin embargo, se prevé que el número de profesionales necesarios capaces de desempeñar las labores de creación o adaptación de contenidos a lenguaje fácil de comprender sea cada vez mayor.

En este contexto heterogéneo y apremiante urge establecer un marco común sobre la práctica actual y la futura formación de profesionales en lenguaje fácil de comprender. Aquí es donde cabe enmarcar el proyecto europeo de innovación docente EASIT (Easy Access for Social Inclusion Training, Formación en Acceso Fácil para la Inclusión Social) ${ }^{2}$, el cual pretende no solo describir este nuevo perfil para dotarlo de visibilidad, sino especialmente proponer para él un programa docente y materiales de formación específicos para quien quiera seguir esta vía de especialización.

2. http://pagines.uab.cat/easit/es [21.05.2019]. 
Así, este artículo define en primer lugar el concepto de lenguaje fácil de comprender y expone sucintamente su alcance en el ámbito hispanohablante. Seguidamente, presenta la metodología aplicada para la recopilación de datos sobre la práctica y la formación actuales en lenguaje fácil de comprender en el marco del proyecto de innovación docente EASIT, basada en un cuestionario cuyos resultados se exponen en el apartado 4. Finalmente, el apartado 5 se plantea, a modo de reflexión final, la necesidad de poner, en adelante, el foco de esta forma de accesibilidad en los medios audiovisuales.

\section{El lenguaje fácil de comprender en lengua española}

Para los propósitos del proyecto EASIT, el lenguaje fácil de comprender se define como aquel que contribuye a hacer el contenido o la información accesibles al máximo número de personas posible. Este concepto bebe de los principios del LL, también llamado lenguaje sencillo o lenguaje claro, y del lenguaje de LF. El LL halla su origen en los movimientos Plain English y Plain Language que tuvieron lugar en los años setenta, cuyo objetivo era denunciar y combatir la dificultad con la que se encontraban los ciudadanos a la hora de acceder a textos de los ámbitos de la jurisprudencia, la administración y la legislación (Bailey 1990). Tal como lo define Badía (2011a), el LL busca "la claridad, la precisión, la concisión" y se trata de una modalidad de lenguaje que cobra importancia en el contexto español a partir de los años ochenta (Badía 2011b). Se ha incidido repetidamente en el hecho de que no se trata de un lenguaje simplificado, sino que exige un esfuerzo. Los expertos han querido distinguirlo del lenguaje de LF, aunque ambos tipos de lenguaje comparten el objetivo de facilitar el acceso a los contenidos.

La IFLA (2012: 5) propone dos definiciones sucintas para la LF, así como un resumen de sus objetivos:

Una significa adaptación lingüística de un texto que facilita más la lectura que un texto medio, pero que no facilita la comprensión; la otra definición es adaptación que hace más fácil tanto la lectura como la comprensión.

El objetivo de las publicaciones de lectura fácil es presentar textos claros y fáciles de comprender apropiados para diferentes grupos de edad. Para alcanzar tales productos, el escritor/editor debe tener en cuenta el contenido, el lenguaje, las ilustraciones y la maquetación. 
Así, la LF no solo comparte el objetivo de claridad con el LL, sino que incide en aspectos de diseño más allá de la redacción, tal como se preveía desde los inicios del movimiento LL (Mazur 2000: 27). Ambos tipos de lenguaje son, por tanto, de interés para nuestro proyecto de investigación.

A modo de guía para los profesionales, desde el año 2018, en español contamos con la primera norma técnica sobre Lectura Fácil, UNE 153101:2018 EX, la cual "detalla las pautas y recomendaciones tanto para la redacción del texto como para el diseño de un documento" (Delgado \& Rodríguez Rubio 2018). De acuerdo con el informe elaborado por Carretero et al. (2017: 17-21) sobre buenas prácticas en lenguaje claro, entre los países de habla hispana donde más se ha trabajado esta cuestión destacan Argentina, Chile, Colombia, España y México. En la mayoría de los casos, las buenas prácticas han pasado por la organización de seminarios de formación y proyectos orientados a mejorar la comunicación entre la administración pública y los ciudadanos. En el caso de España, destaca asimismo la organización social Instituto Lectura Fácil $^{3}$, cuyas funciones, a través de dos estructuras jurídicas diferenciadas, una asociación y una cooperativa de segundo grado integrada por dieciocho entidades, van desde validar y crear documentación escrita en lenguaje de LF hasta incidir en la esfera política, en la investigación y en la sensibilización de los ciudadanos.

Cabe destacar, asimismo, la labor que llevan a cabo distintas asociaciones que pertenecen a la red internacional Easy To Read y trabajan en distintas comunidades autónomas. Por ejemplo, la Associació de Lectura Fàcil, en Cataluña, ofrece un catálogo de obras literarias escritas en lenguaje fácil de comprender que permite filtrar los resultados por lengua, incluida la española ${ }^{4}$. Distintos países hispanohablantes han elaborado igualmente manuales de redacción que facilitan el trabajo de los profesionales más allá de las pautas disponibles - uno de los pioneros en proponer obras de este tipo fue México con su Manual de Lenguaje Claro (2007) 5 - En algunas ciudades, como Madrid, los ayuntamientos han impulsado medidas para favorecer la

3. <http://www.institutolecturafacil.org > [28.06.2019]

4. <http://www.lecturafacil.net/search/> [28.06.2019]. En el momento de redacción de este artículo, el catálogo cuenta con 136 obras adaptadas a LF en español.

5. Badía (2011b) proporciona un listado de recursos útiles en este sentido. 
supresión de barreras cognitivas en los procesos de comunicación entre la Administración y los ciudadanos (Montolío \& Tascón 2017).

Otra prueba reciente de que el lenguaje fácil de comprender cobra relevancia en el ámbito de la lengua española fue la celebración en 2017 de un seminario titulado "Lenguaje claro: reto de la sociedad del siglo XXI", en el que periodistas y escritores de renombre debatieron exclusivamente sobre en qué consiste comunicarse de manera accesible y cuáles eran los principales desafíos para el futuro cercano. Una de las conclusiones destacadas del encuentro fue la necesidad de "profesionalizar el proceso [comunicativo] con la participación de personas especializadas que conozcan las técnicas y pautas" (Fundación San Millán de la Cogolla y Fundéu BBVA 2017: 1).

Sin embargo, hasta donde hemos sido capaces de ver, los programas de formación reglada en lengua española no incluyen contenidos específicos sobre este tema. Asimismo, hay posgrados completos sobre accesibilidad y diseño universal que no abordan este tema. Quien quiere formarse en este sentido cuenta, a lo sumo, con un apartado que introduce a los fundamentos del lenguaje de LF dentro de un módulo más general sobre accesibilidad —así ocurre, por ejemplo, en el caso del programa llamado Experto en Accesibilidad (Instituto Superior de Estudios Lingüísticos y Traducción)—.

Así, pues, de momento y como comprobaremos en los siguientes apartados de este artículo, la especialización y profesionalización esperables de los expertos actuales en la elaboración o adaptación de contenidos fáciles de comprender pasa por la participación en talleres y seminarios que, en aproximadamente la mitad de los casos, son de menos de 30 horas de duración. Por lo general, estos talleres son impulsados por asociaciones como las que hemos mencionado arriba y consisten en cursos breves en los que se practican las técnicas de redacción, sobre todo, en lenguaje de LF. Cabe añadir que, de hecho, la formación que ofrecen estas organizaciones también va más allá de los aspectos de redacción o maquetación que nos ocupan en nuestro proyecto y puede dirigirse a personas interesadas en dinamizar, por ejemplo, un club de lectura de textos fáciles de comprender. 


\section{Metodología}

En este artículo se presentan los resultados de un cuestionario elaborado y distribuido en el marco del proyecto de innovación docente EASIT. Como se ha apuntado en la Introducción, se trata de un proyecto pionero en la elaboración de materiales formativos para un perfil profesional emergente en el ámbito de las lenguas de Europa: el experto en la creación, revisión y adaptación de contenidos fáciles de comprender. A lo largo del proyecto se prevén distintas actividades de investigación —entre las cuales, cuestionarios, grupos de discusión o entrevistas- que aporten información relevante para la elaboración de dichos materiales formativos. El cuestionario que aquí nos ocupa era la primera de ellas.

El objetivo del cuestionario era recabar datos actualizados sobre prácticas y formación actuales en torno al lenguaje fácil de comprender. Los informantes eran personas consideradas expertas en el ámbito. Se diseñó un primer borrador de cuestionario que fue evaluado por todas las entidades participantes en el proyecto y a partir del cual se hicieron pruebas piloto. El cuestionario final ${ }^{6}$ contó con cincuenta y cuatro preguntas, organizadas en cuatro grandes bloques, a saber: a) perfil demográfico, b) estudios y experiencia previa, c) actividad actual, y d) competencias y habilidades. Las preguntas planteadas respondían al estado de la cuestión de acuerdo con la bibliografía especializada consultada. En la mayoría de casos, los participantes tenían la opción de elegir más de una respuesta. Al final de cada apartado, se puso a disposición de los participantes una casilla para aclaraciones u otros comentarios.

La redacción de las preguntas se llevó a cabo en inglés; a continuación, el cuestionario se tradujo a todas las lenguas del proyecto siguiendo los principios del lenguaje fácil de entender. La herramienta empleada para el diseño y distribución del cuestionario fue Web Survey Creator. Los participantes necesitaron 20 minutos aproximadamente para responder las preguntas, y no obtuvieron ninguna compensación por su participación. Los canales de difusión del cuestionario fueron las redes sociales del proyecto y de sus investigadores, así como el contacto directo con expertos reconocidos del ámbito. Se compartió con la Asociación de Lectura Fácil, entidad que tiene potestad

6. <https://ddd.uab.cat/record/203967\&gt> [21.05.2019]. 
en España para certificar el material publicado como acorde a los principios de la LF. En total, el proyecto obtuvo 128 respuestas, 26 de las cuales fueron de expertos que trabajan con la lengua española. Si bien abordamos este estudio desde un punto de vista esencialmente cualitativo, cabe señalar que la cantidad de participantes para el español fue la segunda más amplia, después del alemán (28) y seguida de cerca por el sueco (25). Este dato preliminar de participación nos indica que tratar este tema es especialmente importante en el contexto de la formación en español, ya que nos demuestra que, efectivamente, existe un mercado laboral emergente para esta lengua.

La elaboración y distribución de los cuestionarios cumplió con los procedimientos de ética actuales tal como se describen en Orero et al. (2018). Asimismo, se obtuvieron permisos del comité ético de la Universitat Autònoma de Barcelona para llevar a cabo la investigación.

\section{Resultados}

En este apartado presentamos los resultados del cuestionario aplicado a los expertos cuya lengua principal o única de trabajo es el español. En concreto, nos centramos en los perfiles educativos de los que a día de hoy son considerados expertos en lenguaje fácil de comprender en el ámbito de la lengua española (4.1), así como en sus prácticas actuales en el mundo profesional (4.2). Asimismo, explicamos qué habilidades y competencias se perciben como indispensables o deseables en estos nuevos profesionales, desde el punto de vista de los expertos (4.3). Finalmente, hacemos alusión a resultados obtenidos para otras lenguas que puedan arrojar luz sobre nuestro foco de interés (4.4).

\subsection{Perfiles educativos de los expertos en lenguaje fácil de comprender}

Todos los participantes tienen como lengua de trabajo su lengua materna, el español, excepto dos: uno señala el gallego como lengua materna y como segunda lengua de trabajo; otro, afirma trabajar también con la lengua inglesa. Las expertas son mayoritariamente mujeres (61,54\%), aunque cabe destacar la presencia de hombres entre los considerados expertos del ámbito, ya que en lenguas como el catalán el total de expertas participantes en el cuestionario son mujeres. La franja de edad más frecuente entre los encuestados son los 
41-50 años (42,31\%), si bien 5 de ellos tienen entre 20 y 30 años, 5 tienen entre 31 y 40, y otros 5, entre 51 y 60 años. La mayoría de ellos cuentan con estudios de educación superior a nivel de grado (38,46 \%), máster (38,46 \%) o doctorado (3,85 \%); 4 de los participantes han cursado estudios de formación profesional.

Las áreas de especialización de los expertos son variadas: el área más compartida es la enseñanza y el aprendizaje (23,08 \%), seguida por áreas como las lenguas y la lingüística (19,23 \%), la comunicación y el periodismo $(19,23 \%)$ o la traducción (15,38 \%). En menor medida, los participantes dicen ser especialistas en los ámbitos del trabajo social y la pedagogía especial y de la psicología, entre otros. La mayoría (76,92 \%) ha recibido asimismo formación específica sobre lenguaje fácil de comprender; en concreto, la formación mayoritaria ha sido en lenguaje de LF (85\%). Solo 2 de los participantes dicen haberse formado en LL, y 3, en ambas modalidades. Dicha formación se ha recibido a partir de sesiones internas en el lugar de trabajo ( $55 \%$ ), de talleres (50\%) y cursos de formación profesional (15\%). 5 de los encuestados afirman ser autodidactas y 4 dicen haber recibido otro tipo de formación, sin especificar. Aunque no todos responden la pregunta sobre la cantidad total de horas de formación, la dedicación de los informantes se distribuye tal como se indica en la Tabla 1.

Tabla 1. Horas de dedicación a la formación en lenguaje fácil de comprender de los expertos

\begin{tabular}{|l|l|}
\hline Menos de 10 horas & $10 \%$ \\
\hline Entre 10 y 30 horas & $35 \%$ \\
\hline Entre 30 y 60 horas & $30 \%$ \\
\hline Más de 60 horas & $25 \%$ \\
\hline
\end{tabular}

Su formación en lenguaje fácil de comprender se ha centrado en contenidos impresos (95\%), aunque 8 de los participantes dicen haberse formado también sobre contenidos digitales. Un participante ha recibido formación sobre contenido de audio y otro sobre contenido audiovisual. 2 afirman haber recibido una formación más generalista, no focalizada en ningún tipo de contenido en concreto. En cuanto al ámbito de especialización dentro del lenguaje 
fácil de comprender, la mayoría afirma haberse dedicado a ámbitos variados (65\%), si bien los dos focos de formación parecen haber sido la administración pública y la justicia — por ejemplo, el trabajo con documentos institucionales y administrativos, documentos públicos y jurídicos, declaraciones gubernamentales, contratos, etc.- (35\%) y los medios de comunicación y el periodismo - por ejemplo, noticias, comunicados de prensa, programas de televisión, guiones cinematográficos, contenido en línea, etc.- (también $35 \%$ ). Otros ámbitos de formación especificados por los participantes son la enseñanza ( $25 \%$ ) y la cultura y la literatura (20\%).

En consonancia con el resultado obtenido sobre su formación en LF o LL, las tareas o servicios a los que se han dedicado más esfuerzos durante su formación han sido también en torno a la LF. En concreto, el $75 \%$ se ha formado en adaptación, edición o traducción de textos en LF; el 70 \% afirma haberse formado en la validación y revisión de dichos textos; y el $55 \%$, en su creación. Solo un 20 \% afirma haber recibido formación sobre el control de calidad de textos en LF. Todos los porcentajes, como indicamos, son muy inferiores en relación con las mismas tareas sobre LL.

El tipo de ejercicio que les resultó más útil durante su formación fueron las prácticas y el trabajo junto a expertos (80 \%). También encuentran útiles los ejercicios de escritura ( $75 \%$ ) y revisión (12\%). Todos los que responden a la pregunta sobre la formación en pautas (20) afirman haberse formado siguiendo pautas o recomendaciones de creación. En el 50 \% de los casos se trató de pautas específicas de una lengua, si bien 5 de los informantes dicen haber empleado pautas que no eran específicas de ninguna lengua y 3 , ambos tipos de pautas.

La mayoría de los informantes (85\%) afirma que sigue mejorando sus destrezas trabajando de forma continua en este ámbito. Para su formación continua, sobre todo les resultan útiles el contacto con expertos (75\%), el trato con los usuarios (70\%), el análisis de material actual (50\%) y la participación en nuevas iniciativas de formación (50\%). Un $5 \%$ dice investigar actualmente sobre el tema del lenguaje fácil de comprender. En la casilla de comentarios adicionales, uno de los encuestados subraya la importancia que tiene en este ámbito poner interés en la labor diaria. 


\subsection{Prácticas actuales en el mercado laboral}

Aunque el $70 \%$ de los participantes en el cuestionario recibió un certificado por los estudios cursados que hemos descrito en el apartado anterior, solo uno de ellos indica que se le pidió tal certificado en su lugar de trabajo. Actualmente, estos expertos compaginan diversas tareas en su quehacer profesional: trabajan como formadores $(46,15 \%)$, traductores o adaptadores $(42,31 \%)$, validadores o asesores $(30,77 \%)$, productores, creadores o escritores $(23,08 \%)$, entre otros. Desempeñan su trabajo de forma remunerada en asociaciones sin ánimo de lucro (38,46 \%), en entidades públicas (19,23\%), así como en universidades y centros de investigación (15,38 \%), mayoritariamente. Se trata, en 8 casos, de trabajos a media jornada. Solo uno de ellos trabaja por cuenta propia. Igualmente, solo uno de los participantes afirma trabajar como voluntario para una asociación sin ánimo de lucro.

Se dedican en su mayoría al lenguaje de LF (95\%), solo en 3 casos en combinación con el LL; un resultado que queda acorde con su formación tal como la hemos descrito arriba — solo uno de los encuestados dice trabajar exclusivamente con el LL-. Más de la mitad (55\%) llevan entre cinco y once años en el sector, aunque el carácter emergente de estos profesionales es notorio: 7 de ellos llevan menos de 3 años dedicados al lenguaje fácil de comprender. Todos afirman trabajar con contenido impreso, lo cual de nuevo está en consonancia con su formación, y 8 indican dedicarse también a los contenidos digitales. Los ámbitos para los que crean, revisan o adaptan contenidos son por lo general variados ( $55 \%$ ), si bien los materiales didácticos (45\%) y la documentación jurídica y administrativa (35\%) parecen los contenidos a los que más se dedican.

Al igual que en el caso de la formación, la dedicación a las tareas vinculadas al lenguaje de LF es mayor que la relacionada con el LL: el $70 \%$ de los encuestados valida y revisa textos de LF, el $65 \%$ los adapta edita o traduce, el $45 \%$ los crea o escribe y el $15 \%$ se dedica al control de calidad de este tipo de textos. Es interesante saber que ninguno de ellos realiza estas tareas solo; la Tabla 2 muestra los resultados obtenidos de la pregunta formulada en este sentido. 
Tabla 2. Frecuencia del trabajo en equipo de los expertos en lenguaje fácil de comprender

\begin{tabular}{|l|l|}
\hline La mayoría de las veces trabajo solo & $10 \%$ \\
\hline A veces solo y otras veces en equipo & $30 \%$ \\
\hline La mayoría de veces trabajo en equipo & $25 \%$ \\
\hline Trabajo siempre en equipo & $35 \%$ \\
\hline
\end{tabular}

Además del trabajo con colegas y de la consulta a expertos de fuera del lugar de trabajo, el input de los lectores de contenidos fáciles de comprender cobra gran relevancia en el trabajo de los encuestados: todos afirman estar en contacto con ellos para recibir retroalimentación sobre su producción. El 60 \% dice que este contacto tiene lugar con cada encargo de trabajo, el 15\% afirma que se da a menudo, y el $10 \%$, a veces o pocas veces. La mayoría (70 \%) incorpora a sus textos los comentarios que recibe de los usuarios.

Dos elementos más que los encuestados juzgan útiles son, por una parte, la consulta de otros textos en lenguaje fácil de comprender y, por otra, la familiaridad con las pautas disponibles en la actualidad. Así, en la casilla de comentarios, uno de los participantes añade: «Me inspiro en textos adaptados a lectura fácil de calidad contrastada (por autores de mi confianza». Otro, a su vez, explica: «Viendo otros contenidos cojo ideas de formatos que me gustan (para adaptar/crear mi propia información) y que no me gustan». En cuanto a las pautas, la mayoría $(92,31 \%)$ pone de manifiesto la necesidad de unas pautas compartidas para toda Europa. Por lo general, en su trabajo emplean pautas nacionales (56\%); el $60 \%$ indica, sin embargo, que no utiliza pautas para un idioma concreto.

\subsection{Expectativas sobre el nuevo profesional}

La mitad exacta de los participantes (13) han ejercido o ejercen como formadores de lenguaje fácil de comprender, algunos de ellos esporádicamente. Por este motivo, sus respuestas en cuanto a prioridades en la formación del nuevo experto son muy valiosas para el proyecto EASIT. Una de las preguntas del cuestionario pedía a los participantes que eligieran hasta tres aspectos que considerasen que debían cubrirse en el currículo de un nuevo experto. 
Dos aspectos recibieron una importancia unánime: Por un lado, el 84,62 \% de los encuestados hizo hincapié en el conocimiento de los destinatarios —los tipos de discapacidad, las necesidades de cada grupo, el proceso cognitivo y la percepción-. El 76,62 \%, por otro lado, destacó la relevancia de conocer las recomendaciones y pautas para ejercer la profesión. Otras habilidades, en cambio, relacionadas con la aptitud lingüística o los conocimientos sobre accesibilidad obtuvieron menos del $30 \%$ de votos. La Tabla 3 presenta las respuestas obtenidas en cuanto a los servicios sobre los que valdría la pena incidir en el currículo. De acuerdo con las prácticas actuales, se destaca la importancia de la formación en la validación de textos fáciles de comprender.

Tabla 3. Priorización de servicios en la formación del nuevo experto

\begin{tabular}{|l|c|}
\hline Creación/escritura de contenido fácil de comprender & $19,23 \%$ \\
\hline $\begin{array}{l}\text { Adaptación/edición/traducción de textos fáciles de comprender (es } \\
\text { decir, convertir el texto original en un texto fácil de comprender) }\end{array}$ & $26,92 \%$ \\
\hline Validación/revisión de contenido fácil de comprender & $30,77 \%$ \\
\hline Control de calidad de los textos finales & $19,23 \%$ \\
\hline Otros & $3,85 \%$ \\
\hline
\end{tabular}

Al final del cuestionario, se invitó a los participantes a evaluar la importancia de los siguientes aspectos en relación con la creación de contenido fácil de comprender: (a) diseño y composición de la página, (b) uso fundamentado y consciente del vocabulario, (c) uso de una sintaxis y gramática simples que favorezcan la comprensión del texto, (d) organización clara de la información, (e) multimodalidad, es decir, el uso de diferentes canales para transmitir el significado como, por ejemplo, el uso de texto y vídeo o texto e imágenes. (d) fue el aspecto más valorado unánimemente (92,31\%), seguido de (c) (76,92\%). Cabe señalar, asimismo, que ninguno de los aspectos propuestos fue evaluado como «sin importancia».

Con respecto a cada uno de los aspectos anteriores, se pidió a los informantes concreción sobre los rasgos deseables de una producción de contenido fácil de comprender. A continuación, se resumen los resultados en este sentido: 
- en cuanto al diseño de la página, más de la mitad (57\%) destaca la importancia de la distribución de la información en la página, como, por ejemplo, uso de márgenes y espaciado interlineal amplios, organización en párrafos, posicionamiento de las imágenes, etc.;

- en cuanto al vocabulario, la mitad exacta propone el empleo de voces sencillas y fáciles de comprender, y el 38,46 \% hace hincapié en la importancia de definir las palabras nuevas o difíciles;

- en cuanto a la sintaxis, el 46,15\% propone usar una estructura simple que sea fácil de seguir y leer. Como segundo rasgo relevante, eligen el empleo de oraciones cortas que expresen una idea por frase;

- igualmente, el 46,15\% propone incluir glosarios para facilitar el acceso a la información. El 30,77 \% sugiere ubicar la información más relevante al comienzo del documento;

- aunque no se había otorgado importancia a la multimodalidad en la pregunta sobre habilidades deseables en el futuro experto, una mitad de los encuestados propone como medidas en este respecto usar formatos distintos para transmitir la información y la otra mitad, facilitar la comprensión de las palabras acompañándolas de imágenes grandes.

\subsection{El caso del español en perspectiva: comparación con otras lenguas}

Por último, en nuestra presentación de resultados queremos poner en perspectiva la situación de los expertos que trabajan con la lengua española, cotejándola con algunos de los resultados obtenidos para las demás lenguas del estudio. Así, el primer aspecto que merece la pena destacar es la importancia que cobra entre los expertos en lengua española el input de los usuarios: todos afirman sacarle partido, mientras que en los resultados globales de las encuestas más de un $20 \%$ de los participantes afirmaron pedir la opinión de los usuarios en contadas ocasiones —algunos de ellos, nunca-.

En línea con los resultados globales sobre 128 participantes, la mayoría de expertos denuncia la necesidad de unas pautas compartidas. Este dato es interesante en el sentido de que nos indica que el español no es la única lengua donde los expertos hacen notar la carencia de directrices. De hecho, los resultados globales apuntan a una situación tal vez privilegiada para el español en comparación con otras lenguas: en total, el 40 \% dice emplear 
pautas específicas para su lengua de trabajo. Este porcentaje asciende en un $10 \%$ en el caso del español.

En cuanto a la comparación con lenguas en contacto con el español, como el catalán, es interesante ver que los focos de interés en la formación en lenguaje fácil de entender se distribuyen de forma variada entre los expertos que trabajan con el español (van desde textos del ámbito de la administración pública hasta textos periodísticos). En el caso del catalán, en cambio, el foco recae esencialmente en el ámbito cultural y literario (guías de museos, libretos de ópera, novelas, etc.). Los focos de interés descritos tienen un reflejo patente en la práctica profesional en español, por lo que se confirma la necesidad de formación en todos estos ámbitos.

\section{A modo de conclusión: hora de poner el foco en los contenidos audiovisuales}

Nos encontramos en un momento contradictorio, en el que la legislación regula el derecho de todos los ciudadanos a la información y, sin embargo, gran parte de la información que consumimos no es accesible. Los resultados del cuestionario no dejan lugar a dudas sobre la naturaleza emergente de un nuevo perfil profesional en el ámbito de las lenguas, y en concreto en el de la lengua española, capaz de generar y adaptar contenido a un lenguaje fácil de entender que esté al alcance del mayor número de personas posibles.

Hoy, cuando gran parte del flujo informativo nos llega por medios audiovisuales, nos encontramos con que los expertos en lenguaje fácil de comprender no solo no han recibido formación en este ámbito concreto, y apenas se dedican a él, sino que, entre las habilidades esperables en el nuevo experto, no se otorga apenas importancia a la multimodalidad. Precisamente, el proyecto EASIT busca llenar este vacío. Conocer, a través de los resultados del cuestionario elaborado, los tipos de ejercicios que han sido más útiles en el proceso de formación de los expertos actuales nos brinda una información muy enriquecedora para la creación de unos materiales didácticos verdaderamente útiles para el nuevo experto en accesibilidad capaz de responder a los desafíos de comunicación del mundo actual. 


\section{Referencias bibliográficas}

BADÍA, Javier. (2011a) "Sobre el lenguaje llano." Versión electrónica: <http:// lenguajeadministrativo.com/sobre-el-lenguaje-llano/>

BADíA, Javier. (2011b) "Sobre el lenguaje claro." Versión electrónica: <http:// lenguajeadministrativo.com/sobre-el-lenguaje-llano/>

BAILEY, Edward P. (1990) The Plain English Approach to Business Writing. Oxford: Oxford University Press.

Carretero, Cristina; Jorge Pérez; Luis Lanne-Lenne \& Gema de los Reyes. (2017) Lenguaje Claro. Comprender y hacernos entender. Guía breve. Madrid: Instituto de Lectura Fácil y Red Clarity.

Delgado, Clara Isabel \& Mónica Rodríguez Rubio. (2018) "Primera norma técnica sobre Lectura Fácil." La revista de la normalización española 4. Versión electrónica: <https://revista.une.org/4/primera-norma-tecnica-sobre-lectura-facil.html>

Fundación SAN Millán de la Cogolla y FundÉU BBVA. (2017) "XII Seminario Internacional de Lengua y Periodismo Lenguaje claro, un reto de la sociedad del siglo XXI. Conclusiones." Versión electrónica: <http://www.fundeu.es/ wp-content/uploads/2017/05/Conclusiones_Seminario_Lenguaje_Claro.pdf>

IFLA. (2012) Directrices para materiales de lectura fácil. Versión electrónica: $<$ https://www.ifla.org/files/assets/hq/publications/professional-report/120-es. pdf $>$

MAzUR, Beth. (2000) "Revisiting Plain Language." Versión electrónica: <https:// plainlanguage.gov/resources/articles/revisiting-plain-language/>

Montolío, Estrella \& Mario Tascón (dirs.) (2017) Comunicación Clara: Una herramienta para mejorar la comunicación con la ciudadanía. Guía práctica. Madrid: Ayuntamiento de Madrid.

Orero, Pilar; Stephen Doherty; Jan-Louis Kruger; Anna Matamala; Jan Pedersen; Elisa Perego; Pablo Romero-Fresco; Sara Rovira-Esteva; Olga Soler-Vilageliu $\&$ Agnieszka Szarkowska. (2018) "Conducting experimental research in audiovisual translation (AVT): A position paper." The Journal of Specialised Translation 30, pp. 105-126. 


\section{NOTA BIOGRÁFICA / BIONOTE}

BLANCA ARIAS-BADIA es investigadora posdoctoral Juan de la Cierva en el grupo Transmedia Catalonia (Universitat Autònoma de Barcelona). Es doctora en Traducción y Ciencias del Lenguaje, máster en Literatura Comparada y Traducción Literaria, y licenciada en Traducción e Interpretación por la Universitat Pompeu Fabra. Durante sus estudios realizó estancias en King's College London, University College London y Euskal Herriko Unibertsitatea. Actualmente imparte asignaturas sobre traducción y lingüística aplicada en la Universitat Autònoma de Barcelona, la Universidad Nacional de Educación a Distancia y la Universitat Oberta de Catalunya. Compagina la investigación y la docencia con la traducción literaria y audiovisual.

BLANCA ARIAS-BADIA is a postdoctoral researcher (Juan de la Cierva programme) at Transmedia Catalonia (Universitat Autònoma de Barcelona). She holds a PhD in Translation and Language Sciences, a MA in Comparative Literature and Literary Translation, and a BA degree in Translation and Interpreting from Universitat Pompeu Fabra. In the course of her studies, she has undertaken research stays at King's College London, University College London and Euskal Herriko Unibertsitatea. She is currently lecturing on Translation and Applied Linguistics at Universitat Autònoma de Barcelona, Universidad Nacional de Educación a Distancia and Universitat Oberta de Catalunya. She is also active as a literary and audiovisual translator.

ANNA FERNÁNDEZ-TORNÉ es doctora en Traducción y Estudios Interculturales por la Universitat Autònoma de Barcelona, licenciada en Traducción e Interpretación por la Universitat Pompeu Fabra, máster en Traducción Audiovisual (UAB) y cuenta con un posgrado en Asesoramiento Lingüístico en los Medios Audiovisuales en Catalán (UAB) y el Máster Europeo en Traducción Audiovisual (Università degli Studi di Para). Es profesora del Máster en Traducción Audiovisual de la UAB desde 2006. Ha trabajado como traductora freelance desde 1997, especializándose en la localización de software y de videojuegos, y como traductora audiovisual homologada por Televisió de Catalunya (tanto de alemán como de inglés).

ANNA FERNÁNDEZ-TORNÉ holds a PhD in Translation and Intercultural Studies by the Universitat Autònoma de Barcelona, a European Master's degree in 
Audiovisual Translation by the Università degli Studi di Parma, a Master's degree in Audiovisual Translation and a Postgraduate degree in Linguistic Assessment for the Audiovisual Media by the Universitat Autònoma de Barcelona. She has worked as a freelance translator since 1997, specialising in software and video games localisation, and in audiovisual translation since 2005. She lectures at the Master's degree in Audiovisual Translation at the Universitat Autònoma de Barcelona since 2006. She has worked as a freelance translator since 1997, specializing in the localization of software and videogames, and as an audiovisual translator certified by Televisió de Catalunya (both from German and English). 